\title{
Colémbolos epiedáficos (Hexapoda: Collembola) en dos usos del suelo en la Altillanura colombiana
}

\author{
Dayssy A. Duarte Núñez ${ }^{*}$, Olga P. Pinzón Florián ${ }^{1}$ \& José G. Palacios-Vargas² \\ 1. Facultad de Medio Ambiente y Recursos Naturales, Universidad Distrital Francisco José de Caldas, Bogotá, \\ Colombia; daduarten@correo.udistrital.edu.co,opatriciap@udistrital.edu.co \\ 2. Facultad de Ciencias, Universidad Nacional Autónoma de México, Ciudad de México, México; \\ troglolaphysa@hotmail.com \\ * Correspondencia
}

Recibido 13-VII-2019. Corregido 15-V-2020. Aceptado 25-VIII-2020.

\begin{abstract}
Epiedaphic springtails (Hexapoda: Collembola) in two soil uses of Colombia Eastern plains. Introduction: Epiedaphic springtails participate in a wide variety of services linked to plant nutrient availability, water storage and regulation, soil stability and control of moisture and $\mathrm{pH}$ necessary for soil fertility. Therefore, epiedaphic springtails are sensitive to soil use change. Objective: To compare diversity of epiedaphic springtails in two types of introduced vegetation: pastures and forestations of Eucalyptus pellita in a region of the Colombian eastern plans. Methods: Springtails were collected from introduced pastures and young, middle and mature forested areas of E. pellita, using pitfall traps containing Propylene Glycol (40\%) as preservative, during one dry and one wet season. Alfa and Beta diversity were compared at genus level, and community composition was explained using a set of 14 environmental variables through a redundancy analysis (RDA). Results: Epiedaphic springtails were represented by Entomobryomorpha (62\%), Poduromorpha (17 $\%)$ and Symphypleona (21.1\%), 12 families and 26 genera. Effective number of Collembola genera was lower in plantations but abundance was different only in young plantations during dry season. The variables related to forest coverage, soil organic matter coverage and Nitrogen better explained the observed genera assemblage. Conclusions: Forest plantations allowed an assembly of springtails less diverse than in pastures; but comprised both types of genera, those proper of open areas and those from surrounding natural wooded areas.
\end{abstract}

Key words: bioindicators; effective diversity; forest plantations; land use change; soil fauna.

Duarte Núñez, D.A., Pinzón Florián, O.P., \& Palacios-Vargas, J.G. (2020). Colémbolos epiedáficos (Hexapoda: Collembola) en dos usos del suelo en la Altillanura colombiana. Revista de Biología Tropical, 68(4), 1198-1210.

La Orinoquia colombiana es considerada como una de las regiones biogeográficas con mayor extensión en el país (9238277 ha), la cual alberga una gran diversidad de fauna y flora que cumple un papel fundamental dentro de la interconexión entre ecosistemas. La vegetación de la subregión conocida como altillanura está compuesta principalmente por coberturas de sabanas y pastizales, bosques de galería, morichales y plantaciones forestales (Rippstein,
Escobar, \& Motta, 2001), que proveen una serie de servicios ecosistémicos importantes para el ambiente y la sociedad. No obstante, en las últimas tres décadas en la región se ha acelerado el cambio de las coberturas naturales de sabanas por pasturas, cultivos agrícolas y forestales, ganadería extensiva, explotación de hidrocarburos, entre otras, principalmente en la subregión denominada altillanura bien drenada (Romero, et al., 2009). Cerca del $14 \%$ 
de las sabanas se han transformado durante 20 años con la forestación de dichas áreas para la producción de madera, incrementar la captura de carbono y mejorar las condiciones del suelo como un modelo agroindustrial de desarrollo (Peñuela \& Rodríguez, 2014). Particularmente en el municipio de Villanueva-Casanare, extensiones considerables de plantaciones son establecidas principalmente con especies forestales como Pinus caribaea y Eucalyptus pellita (Refocosta S.A.S, 2019), que junto con la introducción de pasturas han reemplazado las coberturas de sabana intervenidas (Rippstein et al., 2001; Peñuela, Fernández, Castro, \& Ocampo, 2011).

La artropofauna es considerada como elemento fundamental para la provisión de servicios ecosistémicos en un área (Decaëns, Jiménez, Gioia, Measey, \& Lavèlle, 2006). La fauna epiedáfica es sensible a cambios provocados en el suelo por factores ambientales y antrópicos (Uribe et al., 2010) por ello son considerados como bioindicadores de la calidad de los suelos, como es el caso de la clase Collembola (Culik \& Zeppelini, 2003). Los organismos responden de manera diferente ante los disturbios, y aunque se tienen indicios del efecto de algunos cultivos agrícolas, pasturas y perennes que sugieren, que la fauna de lombrices se ve favorecida en las pasturas, pero los demás grupos se ven disminuidos en usos intensivos como las pasturas introducidas y los cultivos, siendo más favorables las coberturas con perennes (Decaëns et al., 2006; Sanabria, Dubs, Lavèlle, Fonte, \& Barot, 2016). Particularmente, las comunidades de carábidos, en la cobertura Eucaliptus pellita en la misma área de este estudio, se encuentran dominadas por especies generalistas cuya diversidad y abundancia ( $q 0$ y $q 1)$ disminuyen en plantaciones de mayor desarrollo (García-Suabita, Pinzón, Spence \& Pinzón- Florián, 2019). La diversidad de Collembola depende de factores como la disponibilidad de materia orgánica y la humedad presente en el suelo (PalaciosVargas, 2000; Socarrás, 2013). Los colémbolos están relacionados con la disponibilidad de nutrientes para las plantas, el almacenamiento y regulación hídrica, la estabilidad de los suelos (mejorando su estructura) y el control de la humedad y $\mathrm{pH}$ necesarios para la fertilidad (Lavèlle et al., 2014).

Los insectos de suelos en la región de la Orinoquia ha sido poco estudiados (MoralesCastaño y Medina, 2009) pero particularmente se desconocen efectos de diferentes usos del suelo sobre la microfauna. De esta manera, se considera que el estudio de la colembofauna presente en el uso anterior y actual del suelo puede contribuir a identificar factores que afectan la diversidad de fauna epiedáfica como lo son la introducción de plantaciones comerciales y su relación directa con el uso del suelo, para de esta manera establecer estrategias integrales de manejo forestal sostenible.

\section{MATERIALES Y MÉTODOS}

Sitio de estudio: El estudio se desarrolló en el municipio de Villanueva en el departamento del Casanare, localizado a 550 m.s.n.m. en las coordenadas (4³9'10.73" N \& 72॰54'58.76" W). El clima está clasificado como tropical monzónico (Am) (García, 2004); presenta un régimen de precipitación monomodal siendo los meses más lluviosos de abril a noviembre y el periodo de sequía entre diciembre y marzo. En promedio la precipitación alcanza 2976 $\mathrm{mm}$ al año, la temperatura media anual es de $26.1^{\circ} \mathrm{C}$ y la humedad relativa es del $76 \%$. La zona de estudio está ubicada en la zona de vida bosque húmedo tropical (bh-T) y presenta suelos de tipo arenoso a franco-arenoso, con fertilidad baja, alta concentración de aluminio y hierro, pobres en fósforo, nitrógeno y materia orgánica (Refocosta S.A.S, 2019).

Muestreo de colémbolos: Con el objeto de comparar la diversidad de colémbolos se muestrearon tres lotes de cada edad de plantaciones jóvenes y adultas de E. pellita $(2,6,8$ y 14 años) con poco manejo (labores silviculturales y culturales), tres bosques de galería y dos lotes con pasturas, para un total de seis coberturas comparadas en 17 unidades de muestreo. El estudio se ejecutó durante los meses 
de noviembre 2014 (época seca) y mayo 2015 (época húmeda). No se reemplazaron bosques naturales para establecer las plantaciones, la inclusión de la diversidad de los bosques de galería es solamente de referencia. En cada uno de los lotes se delimitaron 3 transectos en banda de $50 \mathrm{~m}$ de largo por $3 \mathrm{~m}$ de ancho con una distancia de separación de $20 \mathrm{~m}$ entre cada transecto. Dentro de cada transecto se delimitaron 5 sub-transectos de $10 \mathrm{~m}$ de largo por $3 \mathrm{~m}$ de ancho, correspondiente a la distancia de siembra de la plantación y en cada uno se instaló una trampa de caída (Moreira, Huising, $\&$ Bignell, 2012). Cada trampa consistió en un recipiente plástico con capacidad de $500 \mathrm{~cm}^{3}$ enterrado al límite de la superficie del suelo y protegido de la lluvia en el cual se utilizaron $200 \mathrm{ml}$ de líquido preservante (consistente de una solución de Propilenglicol al 40 \%, Spence $\&$ Niemelä, 1994). Se instalaron un total de 255 trampas de caída (por época de muestreo) y se analizaron las 3 trampas centrales de cada transecto, es decir se evaluaron únicamente un total de 153 trampas por cada época.

Con el objeto de explicar la composición de la colembofauna en función de variables ambientales, se incluyeron variables físicoquímicas del suelo y variables dasométricas de las plantaciones. A cada uno de los árboles de la línea central del transecto se le determinó el diámetro a la altura del pecho (DAP) con una cinta diamétrica y la altura total con un hipsómetro Vertex IV (Haglof®, Langsele, Sweden). Se estimó el porcentaje de cobertura de dosel promedio realizando cuatro medidas sobre cada una de las trampas instaladas con un densitómetro convexo (Forestry Suppliers, Inc., Jackson, MS, USA). Además, se midió la altura de la acumulación de materia orgánica enterrando una escala métrica hasta alcanzar la superficie del horizonte A del suelo en tres puntos a cada lado de cada trampa. También se estimó el porcentaje de área sin cobertura (vegetal o por acumulación de materia orgánica) en el cuadro circundante de cada trampa, equivalente a los cuatro árboles más cercanos sembrados a distancia de $3 \times 3 \mathrm{~m}$ (correspondiente a la distancia de siembra). Por otra parte, en campo se determinó la humedad, conductividad volumétrica y la temperatura del suelo al lado de cada trampa de caída utilizando un reflectómetro (TDR; ICT®) y termómetros de suelo (Amprobe ${ }^{\circledR}$, Everett, WA, USA). Asimismo, se recolectaron $784 \mathrm{~cm}^{3}$ de suelo del centro de cada transecto referenciado y posteriormente, se realizó el análisis físico-químico de la siguiente manera: el porcentaje contenido de humedad se determinó mediante el cálculo de diferencia de pesos húmedo y seco de las muestras, el pH se determinó con ayuda de un potenciómetro, el potasio y el calcio mediante el método de extracción usando NH4-Acetato $1 \mathrm{M} \mathrm{pH} 7$ y posteriormente analizado con absorción atómica, el fósforo disponible se determinó mediante el método Bray II por colorimetría. El porcentaje de carbono orgánico se procesó mediante el método Walkley Black por colorimetría, el porcentaje de nitrógeno mediante el método volumétrico Kieldahl, y finalmente la textura se determinó mediante el método de Bouyoucos (valoración densimétrica por dispersión).

Selección, identificación y análisis de muestras: Para la selección de los colémbolos recolectados se tomó una sub-muestra del 50 $\%$ del total de los individuos mismos que se identificaron con ayuda de un estereoscopio. Previamente, se realizó el proceso de aclarado y montaje según la metodología propuesta por Palacios-Vargas y Mejía-Recamier (2007). Adicionalmente, se llevó a cabo la clasificación morfológica con ayuda de un microscopio de contraste de fases y posteriormente con ayuda de textos de referencia y claves de identificación de géneros (Palacios-Vargas, 1990; Díaz Aspiazu, González Cairo, Palacios-Vargas, \& Luciañez-Sánchez, 2004; Ospina, Serna, Peñaranda, \& Serna, 2003; Palacios-Vargas \& Peñaranda-Parada, 2005; Ávila \& Jaramillo, 2009; Ospina \& Palacios-Vargas., 2009; Bellinger, Christiansen, \& Janssens, 1996).

Análisis de datos: La riqueza de las especies presentes se determinó utilizando la metodología de rarefacción basada en cobertura 
para los órdenes de diversidad $q 0, q 1$ y $q 2$ (Chao et al., 2014), los cuales se relacionan directamente con la riqueza (se analiza bajo la importancia de especies poco comunes), el índice de Shannon (donde se relaciona el peso equivalente entre las especies teniendo en cuenta su abundancia relativa) y el índice de Simpson (mayor importancia a especies comunes). Teniendo en cuenta esto, los valores obtenidos en cada orden de diversidad representan el número de especies (o géneros) equivalentes los que se pueden comparar directamente entre sí (Jost, 2006, 2007). La metodología de rarefacción establece valores de riqueza comparables donde se determinan a partir del nivel de completitud del muestreo (el cual se analizó mediante curvas de acumulación de especies) $\mathrm{y}$ adicionalmente, permite establecer intervalos de confianza del $95 \%$ para determinar si existen diferencias significativas entre los valores calculados. El análisis de los datos recolectados se realizó empleando el software $\mathrm{R}$ versión 3.2.5 mediante los paquetes estadísticos Vegan para el análisis de diversidad involucrando variables ambientales (Oksanen et al., 2016). iNEXT para el análisis de riqueza (Hsieh \& Chao, 2016). Vegetarian para determinar la diversidad Alfa y Beta (Charney \& Record, 2012). Labdsv para la ordenación y clasificación de los conjuntos de datos de diversidad identificados (Roberts, 2016) y también Indicspecies para determinar la relación entre presencia y abundancia de géneros de colémbolos encontrados (De Caceres \& Legendre, 2009).

Para establecer diferencias entre los promedios de las variables dasométricas y ambientales evaluadas en cada uno de los sitios y los obtenidos en la plantación se utilizó la prueba no paramétrica Kruskall-Wallis (García-Suabita et al., 2019). Adicionalmente, se analizó el ensamblaje de colémbolos en función de las variables ambientales, donde se implementó un Análisis de Redundancia (RDA) el cual ajusta los datos en un modelo de regresión multivariada mediante técnicas de ordenación. Para el estudio se excluyeron aquellos géneros cuyas muestras tuvieran menos de 10 individuos, y previamente al análisis se eliminaron aquellas variables que evidenciaran una alta correlación (arena, limo, porcentaje de cobertura del dosel, contenido de humedad del suelo, DAP y CICE). Las variables restantes se analizaron mediante el factor de inflación de varianza (VIF) y se seleccionaron aquellas con VIF $<10$ (Hair, Anderson, Tatham \& Black, 1995). Los análisis se realizaron por transecto ya que las muestras de suelo fueron representativas de cada uno de éstos. Igualmente, se analizó la diversidad Beta entre sitios mediante el cálculo de similitud de distancias de Bray-Curtis (Moreno, 2001). La existencia de géneros indicadores se analizó según Dufrêne y Legendre (1997) y De Caceres y Legendre (2009), que tiene en cuenta la probabilidad de encontrar un género en una cobertura determinada y de igual manera en un hábitat específico (especificidad y fidelidad).

\section{RESULTADOS}

En las áreas muestreadas en este estudio, incluyendo el uso anterior (pasturas) y el actual con plantaciones de E. pellita, se recolectaron 1 157 colémbolos, pertenecientes al orden Entomobryomorpha (61.7\%), Poduromorpha (17 \%) y Symphypleona (21.2 \%); 12 familias y 26 géneros en total (Apéndice 1). La mayor abundancia absoluta se encontró en la plantación de 8 años (22.2 \%) y en la cobertura de pastos (20.1\%). Como era de esperarse, se observó mayor abundancia (57\%) en la época húmeda comparado con la época seca $(42.9 \%)$. Por otra parte, los muestreos representaron apropiadamente los géneros esperados en el área de estudio (Apéndice 2 y Apéndice 3).

Riqueza de colémbolos epiedáficos: Durante la época húmeda, la riqueza de colémbolos expresada en géneros poco comunes $(q 0)$ y la abundancia $(q 1)$ fue significativamente mayor en los pastos y en las plantaciones de 2 años en comparación con las otras edades de plantación (Fig. 1A). La época seca afectó la riqueza y abundancia de colémbolos en las pasturas en comparación con la misma cobertura en la época húmeda (de 26 géneros encontrados en la época húmeda se redujo 
a 12 en la época seca, en un $53.8 \%$ ) y en las plantaciones hasta 6 años (en las plantaciones de 2 y 6 años la reducción fue de $64.7 \%$ y $25 \%$ respectivamente). Mientras que en las plantaciones de 8 años en adelante no se observó una fluctuación marcada de número de especies o de abundancia, y contrario a lo esperado, en la época seca se observó mayor número efectivo de géneros (Fig. 1B).

Por otro lado, cuando se consideró la riqueza equivalente de géneros del orden $q 2$ (con más peso los géneros comunes), en las plantaciones de 2 y 6 años disminuyó sustancialmente el número de géneros identificados para la época seca en comparación con la misma edad para la época húmeda (60 y 37.5 $\%$ respectivamente, Fig. 1C). Durante la época húmeda la mayor abundancia se observó en las plantaciones de 8 y 14 años (137 y 144 respectivamente), mientras que durante la época seca se observó en los bosques de galería.

Géneros indicadores: El análisis de especificidad y fidelidad permitió establecer los géneros Rastriopes (Börner, 1906), Folsomides (Stach, 1922) y Salina (MacGillivray, 1894) como afines a las coberturas de plantaciones de E. pellita de 8 y 14 años mientras que no se encontraron géneros afines a las edades de 2 y 6 años. Asimismo, Seira (Lubbock, 1870), Entomobrya (Rondani, 1861), Dicyrtomina (Börner, 1903) y Brachystomella (Ågren, 1904) son afines a las pasturas y Ptenothrix, Sphyrotheca (Börner, 1906) e Isotoma (Bourlet, 1839) fueron afines a la cobertura de bosque de galería (Tabla 1).

Similitud de géneros de colémbolos encontrados en las coberturas analizadas: La similitud de colembofauna epiedáfica de las pasturas estuvo representada parcialmente en las plantaciones. La similitud varió entre 41 y $66 \%$ en la época húmeda y entre 51 y $67 \%$ en la época seca. La mayor similaridad se observó en las plantaciones de mayor edad, E. pellita de 14 años (66 \%) y E. pellita de 8 años (67\%). Entre edades de plantación también se observó variación en la similitud, los mayores valores
(79\%) se observaron entre las plantaciones de E. pellita de 8 y 14 años de edad para la época húmeda, y $74 \%$ entre E. pellita de 6 y 14 años de edad en la época seca. La similitud de géneros de colémbolos entre las plantaciones de $E$. pellita y el bosque de galería varió entre un 41
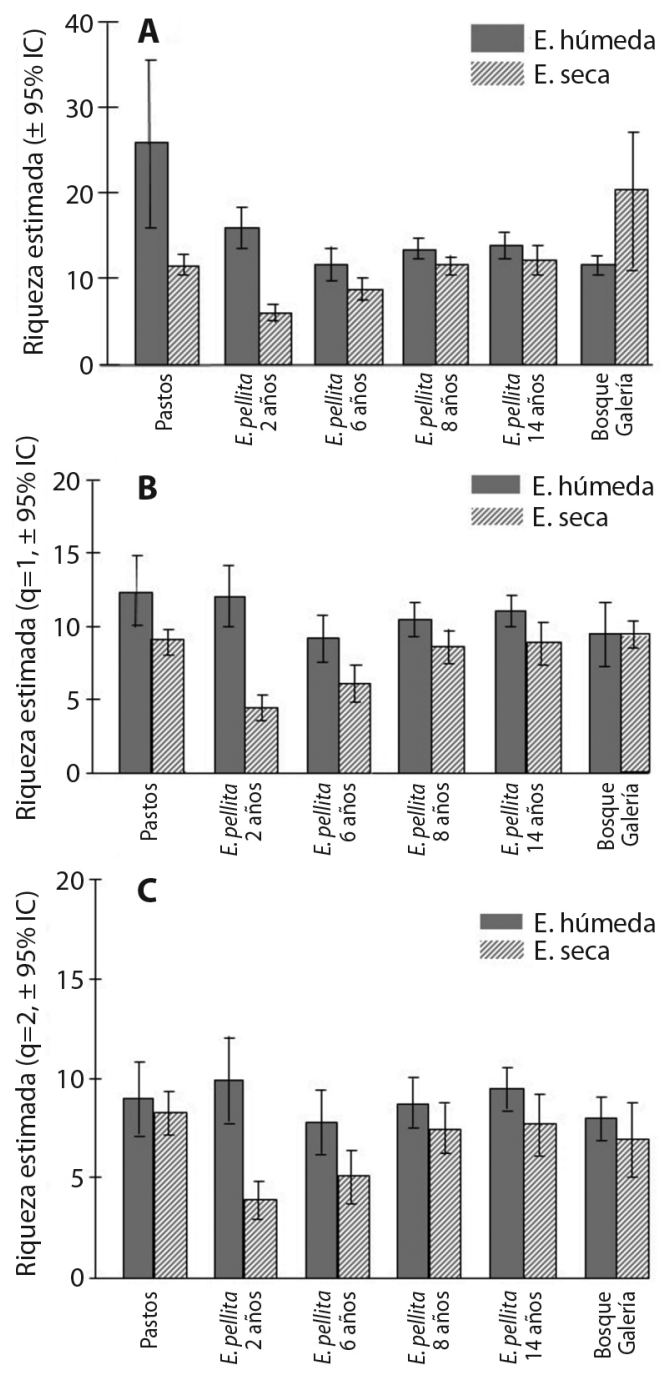

Fig. 1. Número efectivo de géneros de colémbolos epiedáficos por tipo de cobertura y época de muestreo en Villanueva-Casanare. A. Orden de riqueza q0. B. Orden de riqueza q1. C. Orden de riqueza q2.

Fig. 1. Effective number of epiedaphic Collembola genera by type of coverage and sampling season in VillanuevaCasanare. A. Richness order q0. B. Richness order q1. C. Richness order q2. 
TABLA 1

Géneros afines de colémbolos epiedáficos por tipo de cobertura en Villanueva-Casanare

TABLE 1

Indicator genera of epiedaphic Collembola by type of coverage in Villanueva-Casanare

\begin{tabular}{|c|c|c|c|c|c|c|}
\hline \multicolumn{7}{|c|}{ Géneros afines por tipo de cobertura } \\
\hline Tipo de Cobertura & Género & A & B & Stat. & Probabilidad & $\begin{array}{c}\text { Nivel de Significancia } \\
\qquad(0.05)\end{array}$ \\
\hline \multirow[t]{2}{*}{ E. pellita 14 años } & Rastriopes & 1.000 & 0.333 & 0.577 & 0.04 & $*$ \\
\hline & Folsomides & 0.367 & 0.888 & 0.572 & 0.01 & $* *$ \\
\hline \multirow[t]{2}{*}{ E. pellita 8 años } & Sphaeridia & 0.300 & 1.000 & 0.548 & 0.025 & $*$ \\
\hline & Salina & 0.282 & 1.000 & 0.531 & 0.01 & $* *$ \\
\hline \multirow[t]{5}{*}{ Bosque de Galería } & Ptenothrix & 0.923 & 0.888 & 0.906 & 0.005 & $* *$ \\
\hline & Sphyrotheca & 0.903 & 0.888 & 0.896 & 0.005 & $* *$ \\
\hline & Isotoma & 0.888 & 0.666 & 0.770 & 0.005 & $* *$ \\
\hline & Pseudachorutes & 0.409 & 0.666 & 0.522 & 0.045 & $*$ \\
\hline & Trogolaphysa & 0.240 & 1.000 & 0.490 & 0.020 & $*$ \\
\hline \multirow[t]{6}{*}{ Pastos } & Deuterosminthurus & 0.913 & 0.666 & 0.780 & 0.005 & $* *$ \\
\hline & Dicyrtomina & 0.507 & 1.000 & 0.713 & 0.005 & $* *$ \\
\hline & Entomobrya & 0.477 & 1.000 & 0.691 & 0.005 & $* *$ \\
\hline & Denisiella & 0.705 & 0.666 & 0.686 & 0.005 & $* *$ \\
\hline & Seira & 0.454 & 1.000 & 0.674 & 0.005 & $* *$ \\
\hline & Brachystomella & 0.365 & 1.000 & 0.605 & 0.005 & $* *$ \\
\hline
\end{tabular}

$(\mathbf{A}=$ especificidad / specificity; $\mathbf{B}=$ fidelidad / fidelity; $*=\mathrm{P}<0.05 ; * *=\mathrm{P}<0.01 ; * * *=\mathrm{P}<0.001 ;$ ns $=$ no significativo / not significant).

y $49 \%$ para la época húmeda, mientras que para la época seca entre un 53 y 64 \% (Tabla 2).

Composición de colémbolos en función de las variables de la estructura del bosque y las propiedades del suelo: El modelo de redundancia completo (RDA), explicó un $45 \%$ de la varianza observada en la abundancia de colémbolos $\left(\mathrm{R}^{2}=0.26, \mathrm{~F}=5.31, \mathrm{P}=0.001\right)$. De las 14 variables ambientales analizadas (porcentaje exposición del suelo alrededor de la trampa, acumulación de materia orgánica, altura de los árboles, temperatura del suelo, $\mathrm{pH}$, humedad volumétrica, conductividad volumétrica, fósforo, carbono, nitrógeno, aluminio, calcio, potasio y textura), tres se relacionan con la diversidad de colémbolos observada: la exposición del suelo alrededor de la trampa (ESAT), la altura de la vegetación (ALTL) y el contenido de Nitrógeno $(\mathrm{N})$ en el suelo (Apéndice 4). En el primer eje $\left(\mathrm{RDA}_{1}\right)$ se observan dos gradientes representados por el Nitrógeno ( $\mathrm{F}=8.67, \mathrm{P}=0.001)$, donde Ptenothrix está relacionado con altos contendidos de $\mathrm{N}$ en el suelo, lo cual diferencia al bosque de galería de otras coberturas, y para el caso de los pastos, la altura $(\mathrm{F}=4.63, \mathrm{P}=0.001)$ se muestra como una variable inversa. Trogolaphysa y Ptenothrix son los de mayor dominancia para los bosques de galería, mientras Dicyrtomina y Brachystomella son los de mayor dominancia para los pastos. No obstante, en el segundo eje $\left(\mathrm{RDA}_{2}\right)$ se observa la representación del gradiente exposición del suelo alrededor de la trampa ( $\mathrm{F}=2.63, \mathrm{P}=0.010)$ (Fig. 2) relacionados con Lepidocyrtus y Collophora.

\section{DISCUSIÓN}

Este estudio reporta la comparación de la colembofauna observada en cultivos 
TABLA 2

Similitud entre coberturas de pastos, plantaciones de E. pellita y bosque de galería, calculado con el estimador Bray-Curtis

TABLE 2

Similarity between pasture coverings, plantations of E. pellita and gallery forest calculated with Bray-Curtis estimator

\begin{tabular}{|c|c|c|c|c|c|c|}
\hline \multirow{2}{*}{ Cobertura } & \multicolumn{6}{|c|}{ Similitud Bray-Curtis (época húmeda / época seca) } \\
\hline & E. pellita 14 años & E. pellita 8 años & E. pellita 6 años & E. pellita 2 años & B. de galería & Pastos \\
\hline E. pellita 14 años & $0 / 0$ & & & & & \\
\hline E. pellita 8 años & $0.782 / 0.701$ & $0 / 0$ & & & & \\
\hline E. pellita 6 años & $0.414 / 0.733$ & $0.483 / 0.621$ & $0 / 0$ & & & \\
\hline E. pellita 2 años & $0.649 / 0.578$ & $0.615 / 0.573$ & $0.571 / 0.610$ & $0 / 0$ & & \\
\hline B. de galería & $0.454 / 0.579$ & $0.481 / 0.636$ & $0.428 / 0.527$ & $0.405 / 0.619$ & $0 / 0$ & \\
\hline Pastos & $0.653 / 0.640$ & $0.552 / 0.667$ & $0.407 / 0.540$ & $0.527 / 0.515$ & $0.495 / 0.564$ & $0 / 0$ \\
\hline
\end{tabular}

con especies exóticas establecidos en áreas de la altillanura colombiana: pasturas y E. pellita. En general, el número de géneros de colémbolos epiedáficos observado en las plantaciones de E. pellita fue menor y el ensamblaje parcialmente similar al encontrado en las pasturas; sin embargo, sólo se observa menor abundancia de colémbolos en las plantaciones más jóvenes durante la época seca. Por otra parte, se observa un efecto importante de la edad de la plantación y de la época seca. Los ensamblajes de colémbolos encontrados estuvieron relacionados con el contenido de Nitrógeno en el suelo y por el desarrollo de cobertura arbórea (altura de los árboles). Aun cuando los cultivos comparados no reemplazaron los bosques de galería, la colembofauna observada en estas áreas se incluyó para tratar de entender el origen de la composición de la colembofauna encontrada en las plantaciones.

En las pasturas y plantaciones más jóvenes se observó un mayor número de géneros que en las plantaciones de edad media y adulta; sin embargo, al tomar en cuenta la abundancia general y los géneros más comunes ( $q 1$ y $q 2)$ las diferencias se disiparon, especialmente durante la época húmeda. La mayor diversidad de géneros observados en las pasturas puede obedecer a que en éstas se conserven y adapten mejor las especies adaptadas a áreas abiertas propias de las sabanas (uso anterior a los pastos). Teniendo en cuenta que las plantaciones más jóvenes tienen baja cobertura de dosel, la diversidad de áreas abiertas se ve favorecida por un tiempo dentro de la plantación. De manera contraria, en las plantaciones de desarrollo intermedio, escasea la vegetación herbácea y predomina la acumulación de hojarasca del eucalipto, aunque hacia el final de turno y debido a las entresacas, se pueden nuevamente desarrollar algunas herbáceas y arbustos de las familias Melastomataceae, Hypericaceae, Gentianaceae y Lauraceae en plantaciones de E. pellita, las cuales pueden favorecer el incremento de la diversidad de entomofauna al existir una mayor disponibilidad del recurso alimenticio (Fernández Méndez, Camargo Martínez, \& Sarmiento, 2012).

En este estudio, el régimen climático afectó de manera diferente a los colémbolos en las coberturas estudiadas. En general en la época seca, la región alcanza temperaturas extremas (alrededor de los $35^{\circ} \mathrm{C}$, Weather Spark, 2016) y baja humedad en el suelo (Camara, Correia, \& Villela, 2012). Durante la época seca disminuyó significativamente la diversidad y la abundancia de los colémbolos particularmente en las pasturas y las plantaciones de dos y seis años $(q 0, q 1$ y $q 2)$, pero no en las plantaciones de mayor desarrollo. Lo anterior sugiere un efecto benéfico de la cobertura de dosel, posiblemente debido a la regulación de la temperatura del suelo y debido a que dichas coberturas presentan un conjunto heterogéneo de vegetación que satisface las necesidades de la fauna epiedáfica (CONABIO, 2011), que también se observa 

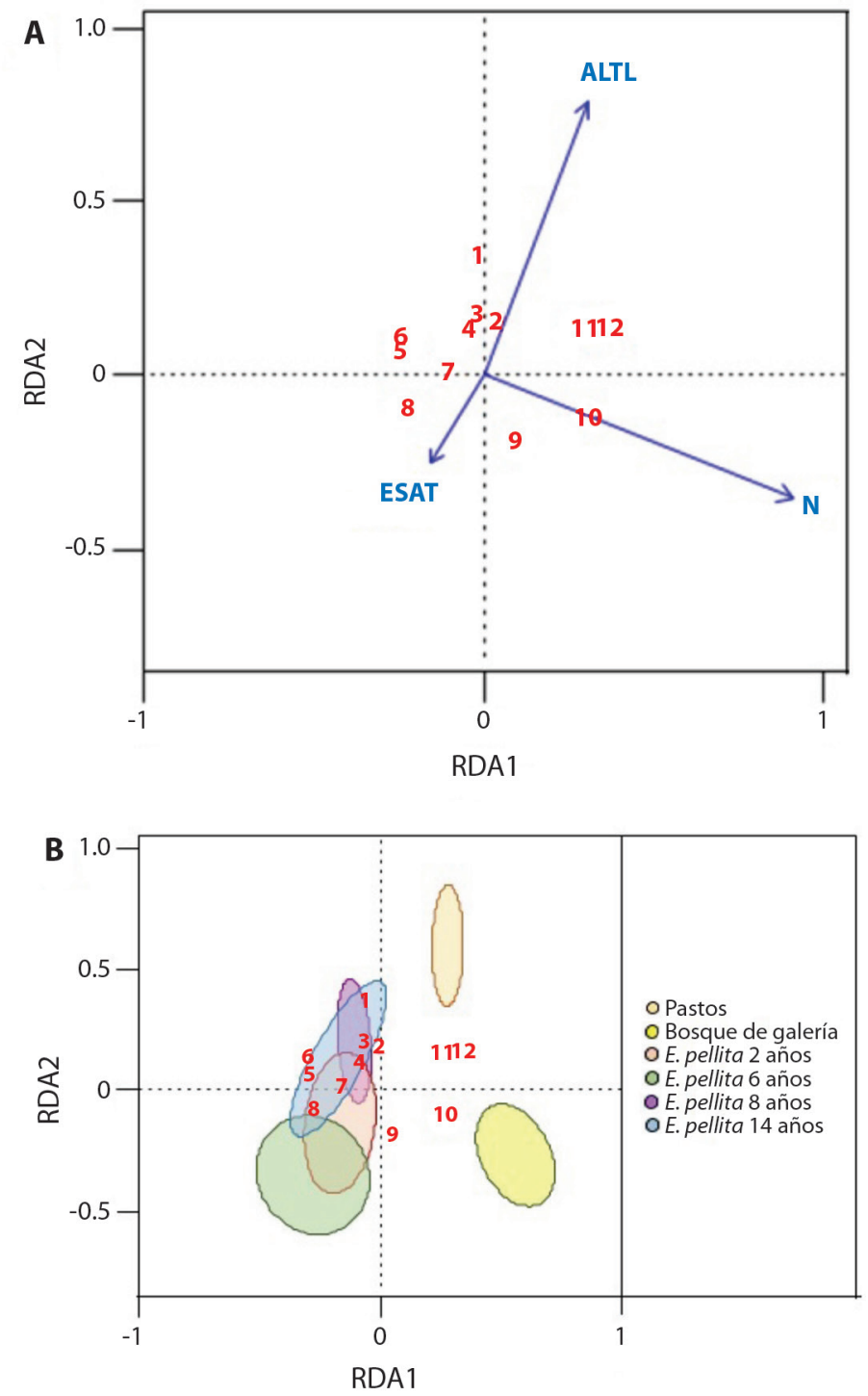

Fig. 2. Diagrama RDA que muestra la composición del ensamblaje de colémbolos en función de las variables de la estructura del bosque y las propiedades del suelo. A. Diagrama de ordenación que muestra la distribución de los géneros de colémbolos en relación con las variables explicativas. B. El mismo modelo con intervalos de confianza del $95 \%$ alrededor de los centroides del grupo superpuestos en el diagrama de ordenación que representa los diferentes hábitats (las elipses sólo se pueden usar como referencia para la interpretación, ya que el tipo de hábitat no se incluyó en el modelo). ESAT: Exposición del suelo alrededor de la trampa, ALTL: Altura del árbol, N: Nitrógeno. 1: Seira, 2: Entomobrya, 3: Cyphoderus, 4: Arlesia, 5: Sphaeridia, 6: Salina, 7: Collophora, 8: Lepidocyrtus, 9: Troglolaphysa, 10: Ptenothrix, 11: Dicyrtomina, 12: Brachystomella.

Fig. 2. RDA ordinations showing assemblage composition of Collembola as a function of forest structure variables and soil properties A. Ordination plot showing distribution of Collembola genera in relation to explanatory variables. B. Same model with $95 \%$ confidence intervals around group centroids overlaid onto the ordination plot representing the different habitats (ellipses may be used only as a reference for interpretation as habitat type was not included in the model). ESAT: Exposure of the soil around the trap, ALTL: Height of trees, N: Nitrogen. 1: Seira, 2: Entomobrya, 3: Cyphoderus, 4: Arlesia, 5: Sphaeridia, 6: Salina, 7: Collophora, 8: Lepidocyrtus, 9: Troglolaphysa, 10: Ptenothrix, 11: Dicyrtomina, 12: Brachystomella. 
en el bosque de galería. Para la época seca se registraron hallazgos de colémbolos que han desarrollado estrategias de adaptación para sobrevivir, como es el caso de Pseudachorutes, Brachystomella y Folsomides, y en general algunos pertenecientes al orden Entomobryomorpha y Poduromorpha (Arbea \& BlascoZumeta, 2001; Guzmán, Melo, Lozano, \& Rivera, 2010).

En las pasturas, las plantaciones y los bosques de galería se observaron géneros indicadores, diferentes para cada tipo de cobertura. Los géneros Seira, Entomobrya, Dicyrtomina y Brachystomella, aunque presentes en todas las coberturas, fueron indicadores de pasturas. La cobertura de pastos favorece la retención de humedad y controla la temperatura del suelo, variables que influyen significativamente sobre la tasa de reproducción de los colémbolos, el crecimiento de los individuos y su distribución a lo largo del perfil (Guzmán et al., 2010), ya que de no existir cobertura vegetal sobre el suelo aumenta la evaporación del agua e incrementa la temperatura, con lo cual se ve afectada directamente la densidad poblacional de los colémbolos, sus ciclos de reproducción y su dinámica poblacional (Cole, Bradford, Shaw, \& Bardgett, 2006). Seira y Entomobrya presentan especies epigeas, adaptadas a áreas abiertas (e.g. sabanas extensivas, Ramos Díaz, Palacios-Vargas, \& Pinzón, en prensa), con adaptaciones morfológicas que los protegen de la radiación solar al reducir la transpiración de la superficie del cuerpo (e.g. escamas, sedas e incluso una pigmentación oscura), así como la existencia frecuente de mudas que les favorecen de condiciones extremas de humedad y temperatura (Guzmán et al., 2010); o incluso a la exposición directa de la lluvia. Dichas adaptaciones resultan favorables en pasturas donde la radiación solar se da de manera directa, al no existir cobertura forestal que proteja al suelo del impacto que pueda ocasionar dicha radiación (Arbea \& Blasco-Zumeta, 2001). El género Brachystomella por su parte tiene hábitos fitófagos micrófagos (son capaces de alimentarse de esporas) y se les encuentra sobre suelos húmedos asociados a hongos y musgos
(Ospina et al., 2003). Los géneros observados en las pasturas en el área de estudio coinciden con reportes en pasturas de otras regiones de Colombia como lo registrado por Ospina et al. (2003), donde se identificaron familias como Hypogastruridae, Brachystomellidae, Neanuridae, Isotomidae, Entomobryidae, Paronellidae, Cyphoderidae, Sminthurididae, Katiannidae, Dicyrtomidae, Bourletiellidae, entre otras.

Por otro lado, los géneros Folsomides y Salina presentaron un alto valor de indicación en las plantaciones adultas. Estos géneros suelen ser abundantes en la vegetación boscosa (Palacios-Vargas \& Castaño-Meneses, 2003), donde sobre las coberturas de dosel maduras se acumula mayor cantidad de materia orgánica y se desarrollan con mayor facilidad hongos y bacterias que hacen parte de la dieta de estos géneros. El tipo de cobertura vegetal presente en un lugar define la abundancia de géneros de colémbolos afines, y específicamente para el caso de plantaciones forestales, la mayor influencia está dada por la edad de los árboles y la profundidad de sus raíces (Palacios-Vargas \& Mejía-Recamier, 2007), además de factores como la calidad del suelo en términos de fertilidad y las condiciones climáticas presentes (Rodríguez Serrano, Acevedo, Álvarez Sánchez, \& Uribe Gómez, 2016). La cantidad y calidad de hojarasca que se acumula en plantaciones adultas es un recurso favorable en la cadena alimenticia para los colémbolos, ya que, durante el proceso de descomposición de la materia orgánica, se favorece la diversidad de hongos y bacterias los cuales son su principal fuente de alimento para los éstos (Guillén, Soto-Adames, \& Springer, 2006). La oferta de materia orgánica disponible produce altos contenidos de ácidos húmicos, disminuyendo la concentración del $\mathrm{pH}$, y generando un ambiente ideal para la reproducción de hongos. Cuando existe una mayor diversidad de hongos se genera un aumento en las asociaciones entre las plantas y las micorrizas y también se incrementa la generación de exudados radicales, los cuales también son una importante fuente de alimento para los colémbolos, los hongos y las bacterias (Guillén et al., 2006). Por esta razón, 
al encontrar una mayor cantidad de materia orgánica en plantaciones adultas se traduce a una mayor oferta de alimento para los colémbolos y su relación con la abundancia presente en dichas coberturas.

Un mayor número de géneros fueron indicadores de bosques naturales, siendo Ptenotrix, Sphyrotheca e Isotoma exclusivos de bosques de galería. Según la literatura (Palacios-Vargas \& Castaño-Meneses, 2003), estos géneros se registran sobre coberturas de dosel maduros y presentan adaptaciones morfológicas para facilitar su movilidad (como la forma del ungues y el apéndice empodial de sus patas), ya que se alimentan de hifas de hongos que crecen sobre la corteza de árboles e incluso se alimentan de musgo y líquenes. Adicionalmente, el desarrollo de dosel presente en el bosque de galería disminuye la radiación solar directa sobre el suelo, manteniendo las condiciones de temperatura bajas y a su vez beneficiando la composición de colémbolos presentes en el suelo (Camara et al., 2012).

Las comunidades de colémbolos en las plantaciones estuvieron compuestas tanto de géneros de áreas abiertas (pasturas), como de bosques naturales. De esta forma, géneros como Seira, Brachystomella y Arlesia pueden encontrarse tanto en pasturas como en plantaciones de E. pellita. Asimismo, se observó similitud entre las coberturas de E. pellita de 14 años y los pastos (60\%) mayor a la similitud entre el bosque de galería y la plantación (40 $\%$ ), posiblemente debido a que a los 14 años de edad de la plantación la densidad de árboles es baja y permite más especies de áreas abiertas, mientras que algunas especies son especialistas del bosque natural y no se adaptan a las plantaciones. Por otra parte, la similitud de géneros entre diferentes edades en las plantaciones es significativa (70-80\%); es decir se encuentran aquellas especies generalistas de áreas abiertas que toleran áreas con dosel arbóreo, y otras generalistas de áreas de bosque que se adaptan a las plantaciones donde se mantienen a lo largo del turno de la plantación.

El modelo RDA, sugiere que la abundancia de Entomobrya responde positivamente al desarrollo de cubierta forestal (altura de los árboles), y se identificó al género Ptenotrix como el de mayor relación con el contenido de Nitrógeno que se encuentra disponible en el suelo en los bosques de galería (es decir la abundancia del género se ve afectada directamente por la disponibilidad de nitrógeno) (Deharveng, 1996), mientras que Lepidocyrtus se vio favorecido con la menor cobertura del suelo (ESAT). En plantaciones jóvenes como es el caso de E. pellita de 2 y 6 años, existe una mayor exposición de suelo descubierto la cual se relaciona fuertemente con los géneros más abundantes, a medida que disminuye la exposición del suelo la abundancia aumenta.

Contrariamente a lo observado en este estudio, en otra región de la altillanura colombiana Ramos Díaz et al. (en prensa), la diversidad de colémbolos aumentó en sabanas forestadas con Acacia mangium, y la abundancia disminuyó con la edad de plantación. Los resultados de ambos estudios coinciden en que la época seca es un factor limitante en la diversidad de colémbolos independientemente de la cobertura vegetal. Lepidocyrtus coincidió como uno de los géneros más abundantes en la época seca en ambos estudios. De la misma forma, según lo encontrado por Ramos Díaz et al. (en prensa), los géneros Salina y Trogolaphysa se afectan por la remoción de la materia orgánica, cambios en el microclima o la reducción de la cantidad y calidad de la hojarasca, lo cual coincide con lo observado para Lepidocyrtus cuando disminuye la disponibilidad de materia orgánica presente en el suelo en este estudio.

La forestación con $E$. pellita genera un hábitat favorable para algunas especies de colémbolos de áreas abiertas y otras de áreas boscosas. Por lo anterior se pueden llegar a considerar como una opción productiva intermedia entre pasturas y bosques naturales y a su vez para mantener, recuperar o incrementar la biodiversidad del lugar (Real de Abreu, Bessão de Assis, Frison, \& Durigan, 2011). Por ser cultivos a largo plazo y por el desarrollo de cobertura de dosel, se considera que las plantaciones forestales pueden ofrecer mayores beneficios que otros cultivos en la sostenibilidad 
del suelo, como ha sido observado en estudios de macrofauna en esta región (Decaëns et al., 2006; Lavèlle et al., 2014; Sanabria et al., 2016), pero para el caso de la microfauna, se requiere optimizar aspectos del manejo forestal, involucrar diferencias entre especies forestales, técnicas de manejo, entre otras (e. g. densidad optima, podas). Finalmente, desde el punto de vista de la diversidad encontrada es importante mencionar que este estudio reporta 27 géneros pertenecientes a 12 familias de la clase Collembola, dentro de los cuales Cyphoderus, Arlesia, Sphaeridia, Collophora, Ptenothrix y Dicyrtomina son considerados como nuevos registros genéricos en la zona de estudio evaluada.

Declaración de ética: los autores declaran que todos están de acuerdo con esta publicación y que han hecho aportes que justifican su autoría; que no hay conflicto de interés de ningún tipo; y que han cumplido con todos los requisitos y procedimientos éticos y legales pertinentes. Todas las fuentes de financiamiento se detallan plena y claramente en la sección de agradecimientos. El respectivo documento legal firmado se encuentra en los archivos de la revista.

\section{AGRADECIMIENTOS}

Los autores agradecen a la empresa Refocosta S.A.S. y particularmente a Guido Gasca y Mónica Sarmiento por el apoyo logístico, la orientación y los permisos para realizar recolecciones dentro de las plantaciones. Los recursos financieros para el muestreo en campo, y procesamiento de muestras en el laboratorio fueron obtenidos con el Convenio 415 de 2013 Colciencias - Universidad Distrital "Francisco José de Caldas". Los autores agradecen a Walter García por el apoyo en los muestreos y registro de variables ambientales en campo, y en el análisis estadístico de los datos. A Adriana Ramos Díaz por el apoyo en la preservación y separación preliminar del material en laboratorio y orientación en la separación preliminar de las muestras. Alejandra Beltrán, Claudia
García y Camila Bautista apoyaron el trabajo de campo, y Jaime Pinzón quien orientó el análisis estadístico de comunidades del proyecto.

\section{RESUMEN}

Introducción: Los colémbolos epiedáficos participan en una amplia variedad de servicios ecosistémicos relacionados con la disponibilidad de nutrientes para las plantas, el almacenamiento y la regulación del agua, la estabilidad del suelo y el control de la humedad y el $\mathrm{pH}$ necesarios para la fertilidad de los suelos. Por esta razón, los colémbolos epiedáficos se consideran como organismos sensibles a los cambios generados en el uso del suelo. Objetivo: Comparar la fauna epiedáfica de dos tipos de vegetación introducida: pastos y plantaciones de Eucalyptus pellita en una región de los Llanos Orientales de Colombia. Métodos: La recolección de los colémbolos se realizó en áreas con cobertura de pastos introducidos y áreas forestadas de diferentes edades, con trampas pitfall que contenían Propilenglicol al $40 \%$ como preservante; durante la época seca y la época húmeda. Se comparó la riqueza efectiva de géneros y se explicó su composición en función de 14 variables ambientales y dasométricas mediante un análisis de redundancia (RDA). Resultados: La colembofauna estuvo compuesta por Entomobryomorpha (62\%), Poduromorpha (17\%) y Symphypleona $(21.1 \%)$ representados en 12 familias y 26 géneros. La riqueza efectiva de géneros fue significativamente mayor en los pastos que en las plantaciones de E. pellita, pero la abundancia no disminuye significativamente, con excepción de las plantaciones jóvenes durante la época seca. El ensamblaje de colémbolos observados en el área se relacionó principalmente con el desarrollo de cobertura forestal, la disponibilidad de materia orgánica alrededor de la trampa y el contenido de Nitrógeno en el suelo. Conclusiones: Las áreas forestadas permitieron un ensamblaje de colémbolos, menos diverso que las pasturas, pero compuesto tanto por especies adaptadas a áreas abiertas, como de las áreas naturales boscosas circundantes.

Palabras clave: bioindicadores; cambio del uso del suelo; diversidad verdadera; entomofauna de suelo; plantaciones forestales.

\section{REFERENCIAS}

Arbea, J. \& Blasco-Zumeta, J. (2001). Ecología de los colémbolos (Hexapoda, Collembola) en Los Monegros (Zaragoza, España). Boletín S.E.A., 28(7), 35-48.

Ávila, D.R., \& Jaramillo, Y.F. (2009). Composición de la clase Collembola en un bosque alto andino de la vereda noruega alta, Silvania, Cundinamarca, Colombia (Tesis Licenciatura). Universidad Distrital Francisco José de Caldas, Bogotá, Colombia. 
Bellinger, P.F., Christiansen, K.A., \& Janssens, F. (1996). Checklist of the Collembola of the World (Base de datos). Recuperado de http://www.collembola.org

Camara, R., Correia, M.F., \& Villela, D.M. (2012). Effects of Eucalyptus plantations on soil arthropod communities in a Brazilian Atlantic Forest conservation unit. Bioscience Journal, 28(3), 445-455.

Chao, A., Gotelli, N.J., Hsieh, T.C., Sander, E.L., Ma, K.H., Colwell, R.K., \& Ellison, A.M. (2014). Rarefaction and extrapolation with Hill numbers: a framework for sampling and estimation in species diversity studies. Ecological Monographs, 84(1), 45-67.

Charney, N., \& Record, S. (2012). Vegetarian: Inext Diversity Measures for Community Data (Version. 1.2., R Package). Recuperado de https://CRAN.R-project. org $/$ package $=$ vegetarian

Cole, L., Bradford, M.A., Shaw, P.J.A., \& Bardgett, R.D. (2006): The abundance, richness and functional role of soil meso and macrofauna in temperate grassland - A case study. Applied Soil Ecology, 33(2), 186-198.

CONABIO. (2011). La biodiversidad en Veracruz: Estudio de Estado. Comisión Nacional para el Conocimiento y Uso de la Biodiversidad. Veracruz, México: Universidad Veracruzana.

Culik, M., \& Zeppelini, D. (2003). Diversity and distribution of Collembola (Arthropoda: Hexapoda) of Brazil. Biodiversity and Conservation, 12, 1119-1143.

Decaëns, T., Jiménez, J.J., Gioia, C., Measey, G.J., \& Lavèlle, P. (2006). The values of soil animals for conservation biology. European Journal of Soil Bio$\log$, 42, 23-38.

De Caceres, M., \& Legendre, P. (2009). Associations between species and groups of sites: indices and statistical inference. Ecology, 90(12), 3566-3574.

Deharveng, L. (1996). Soil Collembola Diversity, Endemism, and Reforestation: A Case Study in the Pyrenees (France). Conservation Biology, 10(1), 74-84.

Díaz Aspiazu, M., González Cairo, V., Palacios-Vargas, J.G., \& Luciañez-Sánchez, M.J. (2004). Clave dicotómica para la determinación de los colémbolos de Cuba (Hexapoda: Collembola). Boletín S.E.A., $34,73-83$.

Dufrêne, M., \& Legendre, P. (1997). Species assemblages and indicator species: the need for a flexible asymmetrical approach. Ecological Monographs, 67(3), 345-366.

Fernández Méndez, F., Camargo Martínez, Y.K., \& Sarmiento, M.B. (2012). Biodiversidad Vegetal Asociada a Plantaciones Forestales de Pinus caribaea Morelet y Eucalyptus pellita F. Muell Establecidas en Villanueva, Casanare, Colombia. Revista Facultad Nacional de Agronomía-Medellín, 65(2), 6755-6770.
García, E. (2004). Modificaciones al Sistema de clasificación climática de Köppen. México: Universidad Nacional Autónoma de México.

García-Suabita, W., Pinzón, J., Spence, J.R., \& Pinzón Florián, O.P. (2019). Epiedaphic ground beetle (Carabidae) diversity in ecosystems transformed by plantations of Eucalyptus pellita in the Orinoco region of Colombia. Neotropical Entomology, 48, 1014-1029.

Guillén, C., Soto-Adames, F., \& Springer, M. (2006). Diversidad y abundancia de colémbolos edáficos en un bosque primario, un bosque secundario y un cafetal en Costa Rica. Agronomía Costarricense, 30(2), 7-17.

Guzmán, C., Melo, O., Lozano, M.D., \& Rivera, F. (2010). Colémbolos (Hexapoda) en un sistema silvopastoril de tres edades de establecimiento y un área arrocera del bosque seco tropical, en el municipio de Piedras, Tolima. Boletín Científico Museo de Historia Natural, 14(2), 155-168.

Hair, J.F., Anderson, R.E., Tatham, R.L., \& Black, W.C. (1995). Multivariate data analysis with readings. USA: Pearson.

Hsieh, K.H., \& Chao, M.A. (2016). INext: An R package for rarefaction and extrapolation of species diversity (Hill numbers). Methods in Ecology and Evolution, 7, 1451-1456.

Jost, L. (2006). Entropy and diversity. Oikos, 113(2), 363-375.

Jost, L. (2007). Partitioning diversity into independent alpha and beta components. Ecology, 88(10), 2427-2439.

Lavèlle, P., Rodríguez, N., Arguello, O., Bernal, J., Botero, C., Chaparro, P., Gómez, Y., Gutiérrez, A., Hurtado, M.P., Loaiza, S., Pullido, S.X., Rodríguez, E., Sanabria, C., Velasquez, E., \& Fonte, S. (2014). Soil ecosystem services and land use in the rapidly changing Orinoco River Basin of Colombia. Agriculture, Ecosystems \& Environment, 185, 106-117.

Morales-Castaño, I.T. y Medina U., C.A. (2009). Insectos de la Orinoquía colombiana: evaluación a partir de la Colección Entomológica del Instituto Alexander von Humboldt (IAvH). Biota Colombiana, 10(1y2), 31-53.

Moreira, F., Huising, E.J., \& Bignell, D.E. (2012). Collembola, acari y otra mesofauna del suelo: el método de Berlese. En A. Karyanto, C. Rahmadi, E. Franklin, F.X. Susilo, \& Wellington, J. (Eds.), Manual de biología de suelos tropicales. Muestreo y caracterización de la biodiversidad bajo suelo (pp. 149-162). México: Secretaría de Medio Ambiente y Recursos Naturales.

Moreno, C.E. (2001). Manual de métodos para medir la biodiversidad. M\&T - Manuales y Tesis Sociedad Entomológica Aragonesa. Zaragoza, España: CYTED, ORCYT-UNESCO, Sociedad Entomológica Aragonesa (SEA). 
Oksanen, O., Blanchet, F.G., Kindt, R., Legendre, P., Minchin, P.R., O'hara, R.B., Simpson, G.L., Solymos, P., Stevens, M.H.H., \& Wagner, H. (2016). Vegan: Community Ecology Package. (Version. 2.3-5., R Package). Recuperado de http://CRAN.R-project.org/ package $=$ vegan

Ospina, C.M., Serna, F.J., Peñaranda, M.R., \& Serna, S.L. (2003). Colémbolos asociados con cultivos de pastos en tres zonas de vida de Holdridge en Antioquia (Colombia). Agronomía Colombiana, 21(3), 129-141.

Ospina, M., \& Palacios-Vargas, J. (2009). A new Denisiella Folsom and Mills, 1938 (Collembola: Sminthurididae) from Colombia. Zootaxa, 2168, 63-68.

Palacios-Vargas, J.G. (1990). El uso de trampas epineústicas para la colecta de microartrópodos. Folia Entomológica Mexicana, 78, 275-277.

Palacios-Vargas, J.G. (2000). Los colémbolos en los ecosistemas mexicanos. Biodiversitas, 29, 12-15.

Palacios-Vargas, J.G., \& Castaño-Meneses, G. (2003). Seasonality and community composition of springtails in Mexican forest. In Y. Basset, V. Novotny, S.E. Miller, \& R.L. Kitching (Eds.), Arthropods of Tropical Forests. Spatio-temporal dinamics and resource use in the canopy (pp.159-169). United Kingdom: Cambridge University Press.

Palacios-Vargas, J.G., \& Peñaranda Parada, M.R. (2005). Two New Species of Paranura (Collembola: Neanuridae) from Colombia and México. Journal of the Kansas Entomological Society, 78(4), 308-314.

Palacios-Vargas, J.G., \& Mejía Recamier, B.E. (2007). Técnicas de colecta, montaje y preservación de microartrópodos edáficos. México: Prensas de Ciencias, Universidad Nacional Autónoma de México.

Peñuela, L., Fernández, A.P., Castro, F., \& Ocampo, A. (2011). Uso y manejo de forrajes nativos en la sabana inundable de la Orinoquía: Sabana inundable. En L. Peñuela (Ed.), Ecosistema estratégico (pp. 66-66). Colombia: La Imprenta Editores S.A.

Peñuela, L., \& Rodríguez, J.J. (2014). Contexto forestal en la cuenca del Río Bita, Vichada: Oportunidades del ordenamiento del Territorio basada en la sostenibilidad. En L. Peñuela (Ed.), Biodiversidad en la cuenca del río Bita (pp. 27-67). Colombia: La Imprenta Editores S.A.

Ramos Díaz, A., Palacios-Vargas, J.G., \& Pinzón, O.P. (en prensa). Diversidad de colémbolos epiedáficos en plantaciones forestales de Acacia mangium y sabanas en la Orinoquía Colombiana. Revista Mexicana de Biodiversidad.

Real de Abreu, R., Bessão de Assis, S., Frison, A., \& Durigan, G. (2011). Can native vegetation recover after slash pine cultivation in the Brazilian Savanna? Forest Ecology and Management, 262(8), 1452-1459.
Refocosta S.A.S. (2019). Resumen Plan de Manejo Forestal 2019 (Reporte técnico). Colombia, Bogotá: Refocosta.

Rippstein, G., Escobar, G., \& Motta, F. (2001). Biodiversidad de la Vegetación de Sabana en la Altillanura Plana y la Serranía de los Llanos Orientales. En G. Rippstein, E. Escobar, J.M. Toledo, M. Fisher, \& E. Mesa (Eds.), Agroecología y biodiversidad de las sabanas en los Llanos Orientales de Colombia (pp. 46-63). Colombia: Centro Internacional de Agricultura Tropical (CIAT).

Roberts, D.W. (2016). Labdsv: Ordination and Multivariate Analysis for Ecology. (Version. 1.8-0., R Package). Recuperado de https://CRAN.R-project.org/ package $=$ labdsv

Rodríguez Serrano, A.C., Acevedo, D.C., Álvarez Sánchez, E., \& Uribe Gómez, M. (2016). Indicadores de calidad de un suelo para la producción de maíz bajo sistemas agroforestal y monocultivo. Revista Mexicana de Ciencias Agrícolas, 16, 3263-3275.

Romero, M.H., Maldonado-Ocampo, J.A., Bogotá-Gregory, J.D., Usma, J.S., Umaña-Villaveces, A.M., Murillo, J.I., Restrepo-Calle, S., Álvarez, M., Palacios-Lozano, M.T., Valbuena, M.S., Mejía, S.L., Aldana-Domínguez, J., \& Payán, E. (2009). Indicadores de seguimiento de la biodiversidad en el área de estudio, a nivel de ecosistemas. En M. Romero-Ruiz (Ed.), Informe sobre el estado de la biodiversidad en Colombia 2007-2008: piedemonte orinoquense, sabanas y bosques asociados al norte del río Guaviare (pp. 15-34). Bogotá D.C., Colombia: Instituto de Investigación de Recursos Biológicos Alexander von Humboldt.

Sanabria, C., Dubs, F., Lavèlle, P., Fonte, S.J., \& Barot, S. (2016). Influence of regions, land uses and soil properties on termite and ant communities in agricultural landscapes of the Colombian Llanos. European Journal of Soil Biology, 74, 81-92.

Socarrás, A. (2013). Mesofauna edáfica: indicador biológico de la calidad del suelo. Pastos y Forrajes, 36(1), 5-13.

Spence, J.R., \& Niemelä, J.K. (1994). Sampling carabid assemblages with pitfall traps: the madness and the method. The Canadian Entomologist, 126(3), 881-894.

Uribe, R., Juárez, C., Montes, M., Palacios-Vargas, J., Cutz, L., \& Mejía, B. (2010). Colémbolos (Hexapoda) como bioindicadores de la calidad de suelos contaminados con hidrocarburos en el sureste de México. Revista Mexicana de Biodiversidad, 81, 153-162.

Weather Spark. (2016). Weather Spark: El clima promedio en Villanueva, Colombia (Base de datos). Recuperado de https://es.weatherspark.com/y/25942/ Clima-promedio-en-Villanueva-Colombia-durantetodo-el-a $\% \mathrm{C} 3 \% \mathrm{~B} 10$ 